\title{
Dosis de N sobre reflectancia espectral, contenido de clorofila y nutrientes en plantas de gerbera
}

\author{
Gabriel A Lorenzo; Libertad Mascarini; Mariel N Gonzalez
}

Universidad de Buenos Aires, Facultad de Agronomía, Cátedra de Floricultura, Buenos Aires, Argentina; lorenzo@agro.uba.ar (autor para correspondencia); lmascari@agro.uba.ar; marielgonzalez2004@yahoo.com.ar

\section{RESUMEN}

En cultivo sin suelo el manejo nutricional debe asegurar la concentración y el balance de los nutrientes, en orden a obtener alta productividad y calidad, a la vez que un ajuste fino de la fertilización contribuirá a reducir el impacto ambiental. Bajo la hipótesis de que hay para gerbera un nivel de $\mathrm{N}$ óptimo que permitirá expresar su máxima productividad potencial, y que a la vez podrá detectarse a través de una técnica no destructiva como la reflectancia del cultivo, así como también si hay variaciones de acuerdo con el momento del año, los objetivos de este trabajo fueron: 1) determinar el efecto de diferentes dosis de $\mathrm{N}$ sobre un cultivo sin suelo de gerbera, 2) detectar patrones de reflectancia espectral que permitan predecir deficiencias nutritivas, y 3) observar si en diferentes momentos del año hay variaciones de la respuesta a las dosis de N. En un cultivo sin suelo de gerbera cv "Sunway" para flor cortada, se ensayaron tres dosis de N, y se registró producción de flores, reflectancia espectral, área foliar y contenido de clorofila y nutrientes en hoja. La producción anual no se diferenció significativamente (110-113 flores/ $\left.\mathrm{m}^{2} / \mathrm{año}\right)$, sin embargo se encontraron diferencias en momentos puntuales en relación con la dosis de $\mathrm{N}$, observándose mejores resultados de la dosis más alta en primavera ( 22 vs. 12 flores $/ \mathrm{m}^{2} / \mathrm{mes}$ ), pero la dosis media mostró mejor respuesta en otoño (12 vs. 5 flores $/ \mathrm{m}^{2} / \mathrm{mes}$ ), mientras que en época invernal no hubo diferencias o bien estas no fueron cuantitativamente importantes ( $2 \mathrm{flores} / \mathrm{m}^{2} / \mathrm{mes}$ ). A partir de la reflectancia se construyeron modelos que permitieron estimar el contenido de clorofila, de nitrógeno y el índice de área foliar (IAF) en forma no destructiva. El IAF podría servir como índice para el manejo nutricional, buscando mantener un IAF del orden de 3, 4 y $2 \mathrm{~m}^{2}$ hoja $/ \mathrm{m}^{2}$ suelo en otoño, invierno y primavera respectivamente.

Palabras clave: Gerbera jamesonii, nitrógeno, radiómetro multiespectral, cultivo sin suelo.

\begin{abstract}
Effect of $\mathrm{N}$ doses on spectral reflectance, chlorophyll content and nutrients on gerbera plants

In soilless crop, nutritional management must ensure the concentration and balance of nutrients, in order to obtain high productivity and quality, while fine adjustment of fertilization contributes to reduce environmental impact. Under the assumption that (1) an optimum level of $\mathrm{N}$ can be determined for gerbera, which provides the maximum potential productivity, (2) that the $\mathrm{N}$ level can be detected through a nondestructive technique, such as crop reflectance, and (3) that variations occur according to season, the objectives of this study were 1) to determine the effect of different doses of $\mathrm{N}$ on soilless-grown gerbera, 2) to detect patterns of spectral reflectance which could predict nutritional deficiencies, and 3) to detect variations of response to $\mathrm{N}$ dose in different season. In a soilless gerbera cv "Sunway" cut flower crop, three doses of $\mathrm{N}$ were tested, and flower production, spectral reflectance, leaf area, nutrient and chlorophyll content in leaf were recorded. The annual production did not differ significantly (110-113 flowers $/ \mathrm{m}^{2} / \mathrm{yr}$ ), however differences in specific moments in relation to $\mathrm{N}$ dose were found, showing better results for the highest dose in spring ( $22 \mathrm{vs} .12$ flowers $/ \mathrm{m}^{2} / \mathrm{months}$ ), but the medium dose showed better response in the autumn (12 vs. 5 flowers $/ \mathrm{m}^{2} /$ months), while no difference occurred in winter or such differences were not quantitatively important ( 2 flowers $/ \mathrm{m}^{2} / \mathrm{months}$ ). Linear models that were constructed from the spectral reflectance allowed us to estimate the chlorophyll content, nitrogen and leaf area index (LAI) in a nondestructive way. The LAI could serve as an index for the nutritional management, looking to keep about 3, 4 and $2 \mathrm{~m}^{2}$ leaf $/ \mathrm{m}^{2}$ ground in autumn, winter and spring, respectively.
\end{abstract}

Keywords: Gerbera jamesonii, nitrogen, multispectral radiometer, soilless culture.

(Recebido para publicação em 4 de abril de 2016; aceito em 13 de março de 2017)

(Received on April 4, 2016; accepted on March 13, 2017)

$\mathrm{E}^{\prime}$ 1 cultivo sin suelo es un método de producción de plantas que se lleva a cabo sobre sustratos inertes, en el cual parte o todos los nutrientes son aportados en el agua de riego (Savvas, 2003). Se requiere una programación muy ajustada del fertirriego, buscando entregar a la planta una cantidad exacta de agua y nutrientes según la edad, el estado de desarrollo y las condiciones ambientales (Keditsu, 2013). Para la adopción de sistemas que ahorren agua y fertilizantes con aceptable nivel de rendimiento, es necesario conocer los efectos que diferentes dosis de nutrientes tienen sobre el cultivo, para aplicar técnicas sustentables, aumentando la eficiencia en el uso de los recursos, y contribuyendo a reducir el impacto ambiental.

En Argentina se producen anualmente 200 millones de flores cortadas, y una de las especies cultivadas más importantes es la gerbera. En La Plata, principal zona 
de producción de flores cortadas, ocupa el cuarto lugar en superficie cultivada (Cieza, 2014).

El efecto del $\mathrm{N}$ en gerbera aún muestra resultados contradictorios. Según Pimple et al. (2006), quienes ensayaron el efecto de $\mathrm{N}$ y el $\mathrm{P}$ combinados en un amplio rango de dosis desde 0 hasta $15 \mathrm{~g} / \mathrm{m}$, una mayor dosis de $\mathrm{N}$ asegura mayor rendimiento y calidad de flor, pero la vida en vaso llegó a superar los 11 días con dosis de $\mathrm{N}$ bajas, del orden de $5 \mathrm{~g} / \mathrm{m}$, siempre en combinación con dosis de $\mathrm{P}$ crecientes.

Una concentración de nitrógeno de $140 \mathrm{mg} / \mathrm{L}$ en la solución nutritiva aumentó en un $20 \%$ la producción de flores de gerbera, así como también mejoró la uniformidad del producto en términos de largo de tallo y diámetro de capítulo, pero la vida en el florero no mostró diferencias significativas, promediando los 9-11 días comparado con concentraciones de 190 y $80 \mathrm{mg} / \mathrm{L}$. Aún así, se determinó que con menor dosis de $\mathrm{N}$ mayor proporción de tallos duraban en vaso menos de 8 días, aunque esto no se vio reflejado en el valor promedio (Mascarini et al., 2005). Por otra parte, Corrales-González et al. (2016) no encontraron diferencias ni de calidad ni de productividad ensayando cuatro niveles de fertilización nitrogenada, entre 56 y $112 \mathrm{mg} /$ planta.

En otro ensayo con diferentes soluciones nutritivas, se destaca la importancia de considerar no solamente los nutrientes específicos sino también las relaciones entre ellos en el que la solución con mejor resultado en términos de cantidad y calidad de flores contenía una concentración de $\mathrm{N}$ de 150 $\mathrm{mg} / \mathrm{L}$, comparado con otras soluciones con una concentración de $\mathrm{N}$ entre 50 a $210 \mathrm{mg} / \mathrm{L}$ (Sirin, 2011).

Los ensayos de campo proveen información valiosa para evaluar el efecto de determinadas técnicas de manejo. Si estos datos pudiesen obtenerse mediante metodologías no destructivas, de aplicación sencilla y rápida determinación, el investigador contaría con herramientas en tiempo real para realizar correcciones (Mascarini et al., 2006; Sahoo et al, 2015).

El uso de sensores no destructivos puede detectar tempranamente posibles deficiencias o excesos nutricionales, siempre que existan los patrones que vinculen la información de dichos sensores con el funcionamiento de la planta. Por ejemplo la medición del contenido de clorofila mediante la absorbancia en dos longitudes de onda, por medio de un dispositivo SPAD ${ }^{\circledR}$ ha sido utilizado en numerosas especies y condiciones de cultivo (Fenech-Larios et al., 2009; Castillo \& Ligarreto, 2010). Otro tipo de medición no destructiva es a través de la radiación reflejada por el cultivo en distintas longitudes de onda, la precisión de este método depende de encontrar las bandas espectrales que mejor se relacionen con la variable ecofisiológica estudiada, lo cual varía según las especies. La reflectancia permite obtener información del status de nitrógeno en hoja, área foliar y biomasa proporcionando características del estado fisiológico de la planta (Sahoo et al., 2015).

La hipótesis de este trabajo es que hay para gerbera un nivel de $\mathrm{N}$ óptimo que permitirá expresar su máxima productividad potencial y que a la vez podrá detectarse a través de una técnica no destructiva como la reflectancia del cultivo, así como también si hay variaciones de acuerdo con el momento del año.

Los objetivos de este trabajo fueron: 1) determinar el efecto de diferentes dosis de $\mathrm{N}$ sobre un cultivo sin suelo de gerbera, 2) detectar patrones de reflectancia espectral que permitan predecir deficiencias nutritivas, y 3 ) observar si en diferentes momentos del año hay variaciones de la respuesta a las dosis de $\mathrm{N}$ ensayadas.

\section{MATERIAL Y MÉTODOS}

El experimento se realizó en la FAUBA (Facultad de Agronomía, Universidad de Buenos Aires ( $34^{\circ} 35^{\prime} \mathrm{S}$; 58 30'O; $30 \mathrm{~m}$ sobre el nivel del mar). El cultivo fue Gerbera jamesonii cv "Sunway"; los plantines fueron provistos por una empresa privada $\left(\right.$ Florist $^{\mathbb{Q}}$, Holanda), siendo el origen de los mismos la micropropagación agámica. El cultivo se ubicó dentro de un invernadero metálico con una cubierta de polietileno térmico de
$150 \mu \mathrm{m}$ de espesor. La orientación del invernadero era NNO-SSE. Tanto la calefacción como la apertura de ventanas estaban automatizadas, estableciéndose umbrales de $15^{\circ} \mathrm{C}$ y $23^{\circ} \mathrm{C}$ respectivamente. Los datos de temperatura y radiación fueron registrados mediante un data logger (Li-COR 1400, Lincoln, Nebraska, Estados Unidos, 1999), a los efectos de determinar las condiciones ambientales a las que estuvo expuesto el cultivo. El fertirriego fue aplicado mediante goteros tipo espagueti $\left(\mathrm{Netafim}^{\circledR}\right)$ con un caudal de $2 \mathrm{~L} / \mathrm{h}$. El sistema estaba controlado automáticamente por un programador de riego, la cantidad de agua aplicada fue calculada mediante el modelo validado por Mascarini et al (2001), que utiliza el método de Penman-Monteith (1965) modificado por FAO (1998). El cultivo se inició con plantines ex-agar rusticados en origen que se entregaron en bandejas alveoladas de 60 celdas, presentando al momento de plantación 4 hojas y una altura de $8-10 \mathrm{~cm}$. Se plantaron en macetas individuales de $8 \mathrm{~L}$ a una densidad de 7 plantas $/ \mathrm{m}^{2}$, en el mes de febrero, y se utilizó como sustrato perlita agrícola $100 \%\left(\right.$ Perlome $^{\circledR}$; San Antonio de los Cobres; Provincia de Salta; Argentina).

La solución nutritiva normal ( $\mathrm{Nn}$ ) se preparó de acuerdo a lo recomendado por Rogers \& Tjia (1990) y tuvo la siguiente composición (en $\mathrm{mmol} / \mathrm{L}$ ): $\mathrm{N}-\mathrm{NO}_{3}=9,95 ; \mathrm{H}_{2} \mathrm{PO}_{4}=0,5 ; \mathrm{K}^{+}=6,0 ;$ $\mathrm{Ca}^{2+}=1,8 ; \mathrm{Mg}^{2+}=0,45 ; \mathrm{Fe}=0,025$; $\mathrm{Mn}=0,005 ; \mathrm{Zn}=0,035 ; \mathrm{B}=0,02 ; \mathrm{Cu}=$ 0,$00075 ; \mathrm{Mo}=0,0005$. La relación $\mathrm{N}: \mathrm{P}_{2} \mathrm{O}_{5}: \mathrm{K}_{2} \mathrm{O}$ fue en este caso 1:0.25:2. El $\mathrm{pH}$ y condutividad eléctrica (CE) fueron de 5,8 y $1,5 \mathrm{dS} / \mathrm{m}$, respectivamente. Esta solución nutritiva con ligeras variaciones fue utilizada en gerbera por Mascarini et al. (2005), entre otros investigadores que trabajaron con gerbera en cultivo sin suelo, y es la que normalmente se utiliza en condiciones de producción comercial. Se preparó en un tanque de $100 \mathrm{~L}$ utilizando las siguientes sales: $\mathrm{Ca}\left(\mathrm{NO}_{3}\right)_{2}=58,8$ $\mathrm{g} ; \mathrm{KNO}_{3}=54,6 \mathrm{~g} ; \mathrm{KH}_{2} \mathrm{PO}_{4}=6,85 \mathrm{~g}$; $\mathrm{K}_{2} \mathrm{SO}_{4}=4,55 \mathrm{~g} ; \mathrm{MgSO}_{4} 7 \mathrm{H}_{2} \mathrm{O}=15,2 \mathrm{~g}$.

Los tratamientos fueron tres: $\mathrm{Nn}$ (nitrógeno normal ya descripto), $\mathrm{N}+$ 
(nitrógeno alto) y N- (nitrógeno bajo), con mayor y menor concentración de $\mathrm{N}_{-} \mathrm{NO}_{3}{ }^{-}$respecto de la $\mathrm{Nn}(13,6$ $\mathrm{mmol} / \mathrm{L}$ y $5,2 \mathrm{mmol} / \mathrm{L}$, respectivamente) manteniéndose constante $\mathrm{pH}, \mathrm{CE}$ y la concentración del resto de los elementos. Dado que el agua utilizada no contenía cantidades importantes de carbonatos y bicarbonatos, no fue necesario adicionar ácidos en ninguno de los tratamientos. Por otro lado el mantenimiento de la $\mathrm{CE}$ fue posible mediante la adecuada combinación de fertilizantes fuente de $\mathrm{N}$ con distinto índice salino y grado equivalente, incorporando $\mathrm{Mg}\left(\mathrm{NO}_{3}\right)_{2}$ a la formulación y balanceando la cantidad de $\mathrm{KNO}_{3}, \mathrm{~K}_{2} \mathrm{SO}_{4}$ y $\mathrm{MgSO}_{4} 7 \mathrm{H}_{2} \mathrm{O}$. $\mathrm{La}$ relación entre los macroelementos $\left(\mathrm{N}: \mathrm{P}_{2} \mathrm{O}_{5}: \mathrm{K}_{2} \mathrm{O}\right)$ fue $1: 0,16: 1,3$ y $1: 0,5: 4$ en $\mathrm{N}+$ y N-respectivamente. Estos tratamientos fueron aplicados desde el inicio del cultivo. La solución nutritiva se renovó cada 7 a 10 días, de acuerdo con el consumo de la misma, y se controló pH y CE dos veces a la semana. Cada tratamiento tuvo 5 repeticiones de 3 plantas cada una, representando 0,43 $\mathrm{m}^{2}$ de cultivo, en un diseño totalmente al azar.

Para obtener un modelo de ajuste que permitiera estimar el contenido de clorofila real a partir de la medición con el medidor portátil MINOLTA SPAD $502^{\circledR}$ (Osaka, Japón, 2000), se midió la hoja más recientemente aparecida totalmente expandida de varias plantas $(n=20)$ con diferente valor relativo indicado por el instrumento, de las cuales luego se determinó en laboratorio el contenido real de clorofila.

Tres meses después de la plantación, comenzaron las mediciones de reflectancia, con un radiómetro multiespectral CROPSCAN ${ }^{\circledR}$ Inc. (Rochester, Estados Unidos, 2000) la reflectancia espectral de una hasta 7 plantas, representando $1 \mathrm{~m}^{2}$ de cultivo, entre $450 \mathrm{~nm}$ y $1600 \mathrm{~nm}$, y luego se determinó el área foliar real de las mismas mediante un areafolímetro (LI-3100; LI-COR, Lincoln, Nebraska, Estados Unidos, 2000). Luego se utilizaron esos datos para generar un patrón capaz de determinar el índice de área foliar (IAF) en forma no destructiva, estimando la misma a partir de la reflectancia espectral, con la misma metodología aplicada por Mascarini et al. (2006), es decir mediante un modelo de regresión lineal entre el área foliar real y la reflectancia en las bandas de $610 \mathrm{~nm}$ y $780 \mathrm{~nm}$; este modelo se aplicó para estimar el IAF en otros momentos del ciclo de cultivo, de forma no destructiva. De la misma manera se generó un modelo de regresión lineal para estimar el contenido real de clorofila, con el valor de reflectancia en las bandas de $660 \mathrm{~nm}$ y $710 \mathrm{~nm}$. Luego se midió la reflectancia espectral del cultivo en tres momentos.

Para determinar si los tratamientos afectaban la composición tisular de las plantas, se realizaron análisis foliares, en la hoja más recientemente aparecida totalmente expandida, de acuerdo a la metodología propuesta por Jones Junior \& Case (1990) (P por colorimetría, S por turbidimetría, N, K, Ca, Mg, Fe, $\mathrm{Mn}, \mathrm{Zn}, \mathrm{Cu}$ y $\mathrm{S}$ por absorción atómica). Esto se realizó en otoño (abril) y en primavera (septiembre), momentos en que la productividad de flores estaba en aumento.

Las flores fueron cosechadas al alcanzar el estado de madurez comercial, es decir, cuando dos círculos de flores liguladas muestran polen desarrollado (Rogers \& Tjia, 1990). Los resultados fueron analizados mediante ANOVA, test de Duncan para separación de medias y regresión lineal múltiple.

\section{RESULTADOS Y DISCUSION}

\section{Producción de flores}

La producción total de flores considerada en todo el período analizado no mostró diferencias significativas, pero sí se detectaron en ciertos momentos para algunos de los tratamientos (Figura 1a). En Diciembre la producción de $\mathrm{N}$ - fue de un $80 \%$ y un $50 \%$ mayor que en Nn y N+, respectivamente; mientras que en Febrero Nn obtuvo 252\% y 55\% más flores que $\mathrm{N}-\mathrm{y} \mathrm{N}+$ respectivamente. Para el mes de abril los tres tratamientos se diferenciaron, siendo la producción $\mathrm{Nn}$ un $48 \%$ y $254 \%$ mayor que $\mathrm{N}-\mathrm{y}$ $\mathrm{N}+$ respectivamente. En agosto $\mathrm{N}+$ fue significativamente superior siendo $44 \%$ y $27 \%$ que $\mathrm{N}$ - y Nn respectivamente. Las variaciones encontradas a lo largo del año responden sobre todo a los cambios en la radiación global y en menor medida a los cambios de temperatura, propios de diferentes estaciones (Figura 1b). Se observa que los momentos de picos y depresiones fueron poco afectados por las distintas dosis de $\mathrm{N}$, pero sí son coincidentes con la marcha de la radiación global y la temperatura media. Se ha informado para 8 cultivares de gerbera cultivados en condiciones tropicales a lo largo de dos años, que las diferencias no se relacionaban con cambios en la eficiencia fotosintética sino con diferencias varietales, mostrando un patrón de producción similar al encontrado en nuestro trabajo (Jaimez et al., 2013). Si bien está demostrado que un mayor suministro de $\mathrm{N}$ aumentó la tasa fotosintética, esto no se tradujo en un rendimiento en flores significativamente mayor (CorralesGonzález et al., 2016). Es decir, las diferencias puntuales encontradas pueden estar relacionadas con un desfasaje temporal de alocación de materia seca en diferentes órganos, pero considerado a lo largo del año no determina diferencias significativas de productividad.

\section{Análisis foliar}

Se encontraron diferencias en el contenido foliar de $\mathrm{N}$ en otoño, siendo en $\mathrm{N}+21 \%$ y $43 \%$ mayor que $\mathrm{Nn}$ y $\mathrm{N}$ - respectivamente, mientras que en primavera estas diferencias fueron de $17 \%$ y $26 \%$. Además se encontró mayor contenido de $\mathrm{Ca}$ bajo $\mathrm{Nn}$, $244 \%$ y $174 \%$ respecto de $\mathrm{N}-\mathrm{y} \mathrm{N}+$ respectivamente en otoño, mientras que en primavera estas diferencias fueron de $203 \%$ y $136 \%$ respectivamente (Tabla 1a). Los micronutrientes mostraron amplias variaciones y no se encontraron diferencias significativas entre tratamientos. En ambos momentos se detectaron amplias diferencias entre los tratamientos en cuanto a las relaciones elementales entre diferentes nutrientes (Tabla 1b).

El uso de soluciones nutritivas con diferente relación molecular entre elementos podría influir la absorción de cada uno de ellos y por lo tanto conducir a resultados controversiales (Tréjo-Tellez \& Gómez-Merino, 2012), En nuestro caso, a pesar que la concentración de $\mathrm{N}$ 

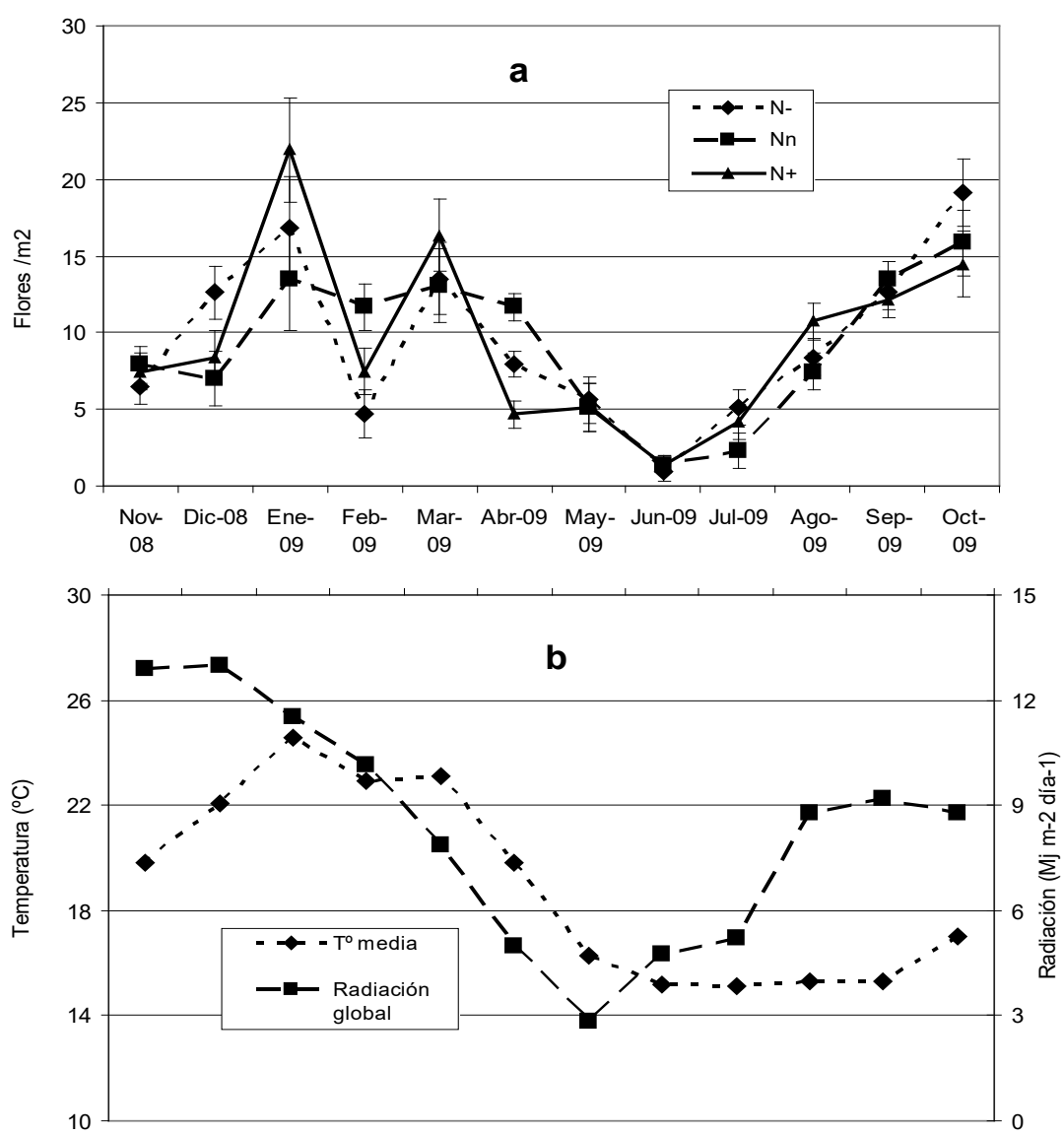

Figura 1. a) Evolución de la producción mensual de flores (flores $/ \mathrm{m}^{2}$ ) de un cultivo sin suelo de gerbera para flor de corte bajo tres niveles de fertilización nitrogenada: $\mathrm{N}-=5,6 ; \mathrm{Nn}=$ 9,95 y N+=13,6 mmol/L N-NO - Las barras verticales indican la DMS (diferencia mínima significativa) a $\mathrm{p} \leq 0,05(\mathrm{n}=45)$. b) Evolución de la temperatura media y la radiación global en el sitio experimental $\left\{\mathrm{a}-=\right.$ Evolution of monthly flower production (flowers $/ \mathrm{m}^{2}$ ) of a gerbera cut flower soilless crop under three levels of nitrogen fertilization: $\mathrm{N}-=5.6 ; \mathrm{Nn}=9.95$ and $\mathrm{N}+=13.6 \mathrm{mmol} / \mathrm{L} \mathrm{N}^{-\mathrm{NO}_{3}}$. Vertical bars indicate LSD (least significant difference) at $\mathrm{p} \leq 0.05$ $(n=45) . b-=$ Evolution of mean temperature and global radiation in the experimental site $\}$. Buenos Aires, FAUBA, 2008.

fue el único elemento distintivo, alteró significativamente la composición de la solución del sustrato y provocó una absorción diferencial de otros nutrientes además, dado que se alteró el contenido de los mismos en hoja. Estos desbalances, más que el contenido absoluto de nutrientes, podrían ser la principal causa de las oscilaciones observadas en la producción de flores.

Reflectancia espectral, clorofila e indice de área foliar

La medición de la reflectancia espectral ofrece un método rápido, confiable y no destructivo para la evaluación de diversas variables ecofisiológicas del cultivo. Además es reflectancia espectral, contenido de clorofila y área foliar, se obtuvieron varios modelos lineales (Tabla 2). Se encontró una regresión linear entre el logaritmo natural del contenido de clorofila y el índice $\operatorname{SPAD}\left(\mathrm{R}^{2}=0,65\right)$. Con los datos de área foliar real y de reflectancia del cultivo, se construyó un patrón para estimar el IAF en cualquier momento, resultando un modelo lineal con las longitudes de onda R780 y R610 $\left(R^{2}=0,77\right)$. Se obtuvo además un modelo lineal entre el contenido de clorofila y la reflectancia en las longitud de onda R710 y el nivel de $\mathrm{N}$ en la solución nutritiva $\left(\mathrm{R}^{2}=0,66\right)$. Por otra parte también se encontró un buen ajuste entre el nivel de $\mathrm{N}$ en la solución nutritiva y la reflectancia en R660 $\left(R^{2}=0,63\right)$. Combinando estos últimos podría estimarse el contenido de clorofila directamente a partir de los datos de reflectancia espectral, tomando los valores en R660 y R710 $\left(\mathrm{R}^{2}=\right.$ $0,59)$, Todos estos modelos resultaron altamente significativos $(\mathrm{p} \leq 0,001)$.

A partir de las mediciones de reflectancia espectral, se estimó el IAF y el contenido de clorofila en hoja en tres momentos del cultivo: otoño, invierno y primavera. Se observa que hubo diferencias encontradas en la reflectancia espectral, y en invierno se observó el mayor IAF. La clorofila fue afectada fuertemente, siendo el invierno la época donde se encontraron las mayores diferencias (Figura 3). En todos los casos, bajo N- se observó menor nivel de clorofila, en otoño y en primavera fue alrededor de $50 \%$ menor que en $\mathrm{Nn}$ y $\mathrm{N}+$ mientras que en invierno también en $\mathrm{N}+$ se observó un menor contenido de clorofila. Estas variaciones responderían a la aclimatación de la planta frente a un ambiente cambiante, como ser diferente calidad y cantidad de luz y estado nutricional. Plantas creciendo en ambientes de menor intensidad lumínica tienden a invertir más en área foliar que en raíces, y además presentan mayor contenido de clorofila (Athanasiou et al., 2010). Aparentemente, habría una reducción en los niveles de ciertos componentes de los cloroplastos, quedando estos recursos disponibles, sobre todo $\mathrm{N}$ orgánico, para su uso en otras partes de 
la planta (Walters, 2005).

Otra cuestión a considerar es la relación $\mathrm{N}: \mathrm{Ca}$, que parece estar relacionada con la aparición de clorosis. Fernández-Falcón et al. (2006) investigaron la variación temporal del nivel nutricional y de clorofila en hojas de Leucospermum cordifolium cv 'High Gold', y determinaron que todos los individuos que presentaban síntomas de clorosis tenían una alta relación $\mathrm{N}: \mathrm{Ca}$ en hoja, aunque desde el punto de vista del contenido de $\mathrm{N}$ todos se encontraban dentro del rango de suficiencia.

En nuestro caso, tanto en otoño como en primavera, las plantas bajo $\mathrm{N}$ y $\mathrm{N}+$ presentaron alrededor del doble de relación $\mathrm{N}$ : $\mathrm{Ca}$ que aquellas bajo
Nn, lo cual explicaría las diferencias encontradas en el contenido de clorofila. El contenido de $\mathrm{Ca}$ foliar en $\mathrm{N}$ - y $\mathrm{N}+$ sería deficiente, mientras que el contenido de $\mathrm{K}$ sería levemente inferior al recomendado en todos los tratamientos, de acuerdo con los rangos de referencia indicados por Jeong et al. (2009).

Tabla 1. a) Contenido de nutrientes y b) relaciones entre nutrientes en la hoja más joven totalmente expandida de un cultivo de gerbera para flor de corte bajo tres niveles de fertilización nitrogenada, en dos momentos del año (a) Nutrient composition, and b) Nutrient ratios of the youngest fully expanded leaf of a gerbera cut flower soilless crop under three levels of nitrogen fertilization in two seasons of the year\}. Buenos Aires, FAUBA, 2008.

\begin{tabular}{|c|c|c|c|c|c|c|}
\hline \multirow{3}{*}{ Tratamientos } & \multicolumn{6}{|c|}{ a) Contenido de elementos en hoja (\% de materia seca) } \\
\hline & \multicolumn{3}{|c|}{ Abril } & \multicolumn{3}{|c|}{ Septiembre } \\
\hline & $\mathbf{N}-$ & $\mathbf{N n}$ & $\mathbf{N}+$ & $\mathbf{N}-$ & Nn & $\mathbf{N +}$ \\
\hline $\mathrm{N}(\mathrm{g} / \mathrm{kg})$ & $1,77 \mathrm{a}$ & $2,10 \mathrm{~b}$ & $2,54 \mathrm{c}$ & $2,19 \mathrm{a}$ & $2,37 \mathrm{~b}$ & $2,77 \mathrm{c}$ \\
\hline $\mathrm{K}(\mathrm{g} / \mathrm{kg})$ & $2,08 \mathrm{a}$ & $2,17 \mathrm{a}$ & $2,16 \mathrm{a}$ & $2,16 \mathrm{a}$ & $2,04 \mathrm{a}$ & $2,04 \mathrm{a}$ \\
\hline $\mathrm{Ca}(\mathrm{g} / \mathrm{kg})$ & $0,45 \mathrm{a}$ & $1,10 \mathrm{~b}$ & $0,63 \mathrm{a}$ & $0,53 \mathrm{a}$ & $1,08 \mathrm{~b}$ & $0,79 \mathrm{a}$ \\
\hline $\operatorname{Mg}(\mathrm{g} / \mathrm{kg})$ & $0,18 \mathrm{a}$ & $0,18 \mathrm{a}$ & $0,14 \mathrm{a}$ & $0,16 \mathrm{a}$ & $0,21 \mathrm{~b}$ & $0,13 \mathrm{a}$ \\
\hline $\mathrm{S}(\mathrm{g} / \mathrm{kg})$ & $0,10 \mathrm{a}$ & $0,12 \mathrm{a}$ & $0,13 \mathrm{a}$ & $0,09 \mathrm{a}$ & $0,08 \mathrm{a}$ & $0,10 \mathrm{a}$ \\
\hline $\mathrm{Fe}(\mathrm{mg} / \mathrm{kg})$ & $0,0553 \mathrm{a}$ & $0,0533 \mathrm{a}$ & $0,0537 \mathrm{a}$ & $0,0570 \mathrm{a}$ & $0,0327 \mathrm{a}$ & $0,0490 \mathrm{a}$ \\
\hline $\mathrm{Zn}(\mathrm{mg} / \mathrm{kg})$ & $0,0049 \mathrm{a}$ & $0,0044 \mathrm{a}$ & $0,0040 \mathrm{a}$ & $0,0057 \mathrm{a}$ & $0,0049 \mathrm{a}$ & $0,0058 \mathrm{a}$ \\
\hline \multirow[t]{2}{*}{$\mathrm{Mn}(\mathrm{mg} / \mathrm{kg})$} & $0,0317 \mathrm{a}$ & $0,0237 \mathrm{a}$ & $0,0377 \mathrm{a}$ & $0,0025 \mathrm{a}$ & $0,0107 \mathrm{a}$ & $0,0323 \mathrm{a}$ \\
\hline & \multicolumn{6}{|c|}{ b) Relaciones elementales en hoja } \\
\hline $\mathrm{N}: \mathrm{K}$ & $0,85 \mathrm{a}$ & $0,97 \mathrm{a}$ & $1,18 \mathrm{~b}$ & $1,01 \mathrm{a}$ & $1,17 \mathrm{~b}$ & $1,36 \mathrm{c}$ \\
\hline $\mathrm{N}: \mathrm{Ca}$ & $4,45 \mathrm{a}$ & $1,91 \mathrm{~b}$ & $4,01 \mathrm{a}$ & $4,25 \mathrm{a}$ & $2,23 \mathrm{~b}$ & $3,50 \mathrm{c}$ \\
\hline $\mathrm{N}: \mathrm{Mg}$ & $10,68 \mathrm{a}$ & $11,90 \mathrm{a}$ & $18,75 \mathrm{~b}$ & $13,76 \mathrm{a}$ & $11,24 \mathrm{a}$ & $21,37 \mathrm{~b}$ \\
\hline $\mathrm{K}: \mathrm{Ca}$ & $5,17 \mathrm{a}$ & $1,97 \mathrm{~b}$ & $3,42 \mathrm{c}$ & $4,19 \mathrm{a}$ & $1,93 \mathrm{~b}$ & $2,58 \mathrm{c}$ \\
\hline $\mathrm{K}: \mathrm{Mg}$ & $12,33 \mathrm{a}$ & $12,27 \mathrm{a}$ & $15,95 \mathrm{a}$ & $13,51 \mathrm{a}$ & $9,60 \mathrm{~b}$ & $15,83 \mathrm{a}$ \\
\hline $\mathrm{Ca}: \mathrm{Mg}$ & $2,42 \mathrm{a}$ & $6,25 \mathrm{~b}$ & $4,70 \mathrm{c}$ & $3,35 \mathrm{a}$ & $5,13 \mathrm{~b}$ & $6,11 b$ \\
\hline
\end{tabular}

$\mathrm{N}-=5,6 ; \mathrm{Nn}=9,95$ y N+=13,6 mmol/L N-NO${ }_{3}^{-}$. Letras diferentes en la misma fila indican diferencias significativas a $\mathrm{p} \leq 0,05(\mathrm{n}=15)$ \{different letters in the same row indicate signficant differences at $\mathrm{p} \leq 0.05(\mathrm{n}=15)\}$.

Tabla 2. Modelos lineales obtenidos a partir de las mediciones de reflectancia espectral, área foliar y contenido de clorofila de un cultivo sin suelo de gerbera para flor de corte de bajo tres niveles de fertilización nitrogenada (linear models produced from measurements of spectral reflectance, leaf area and chlorophyll content of a gerbera cut flower soilless crop under three levels of nitrogen fertilization). Buenos Aires, FAUBA, 2008.

\begin{tabular}{|c|c|c|c|c|}
\hline Variable estimada & Variable medida & Modelo & Significancia & $\mathbf{R}^{2}$ \\
\hline $\begin{array}{l}\text { Contenido de clorofila } \\
(\mathrm{mg} / \mathrm{g})\end{array}$ & Índice SPAD (\%) & Ln clorofila $=-1,5+0,0635 * \operatorname{SPAD}(\%)$ & $* * *$ & 0,65 \\
\hline $\begin{array}{l}\text { Índice de área foliar (IAF) } \\
\left(\mathrm{m}^{2} \text { hoja } / \mathrm{m}^{2} \text { suelo }\right)\end{array}$ & R610, R780 (\%) & $\mathrm{IAF}=1,246 *(\mathrm{R} 780 / \mathrm{R} 610)-3,6261$ & $* * *$ & 0,77 \\
\hline $\begin{array}{l}\text { Contenido de clorofila } \\
(\mathrm{mg} / \mathrm{g})\end{array}$ & $\begin{array}{c}\mathrm{R} 710(\%), \mathrm{N} \\
\left(\mathrm{mmol} / \mathrm{L} \mathrm{N}^{-\mathrm{NO}_{3}^{-}}\right)\end{array}$ & $\begin{array}{c}\text { Clorofila }=7,055+0,259 * \mathrm{~N}- \\
0,250 * \mathrm{R} 710\end{array}$ & $* * *$ & 0,66 \\
\hline 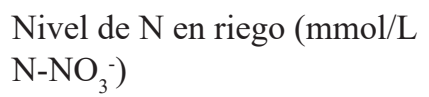 & R660 (\%) & $\mathrm{N}=0,751+1,428 * \mathrm{R} 660$ & $* * *$ & 0,63 \\
\hline $\begin{array}{l}\text { Contenido de clorofila } \\
(\mathrm{mg} / \mathrm{g})\end{array}$ & R660, R710 (\%) & $\begin{array}{c}\text { Clorofila }=8,562-0,254 * \mathrm{R} 710+ \\
0,139 * \mathrm{R} 660\end{array}$ & $* * *$ & 0,59 \\
\hline
\end{tabular}

$\mathrm{N}-=5,6 ; \mathrm{Nn}=9,95 ; \mathrm{N}+=13,6 \mathrm{mmol} / \mathrm{L} \mathrm{N}-\mathrm{NO}_{3} ; * * *: \mathrm{p} \leq 0,0001$. 

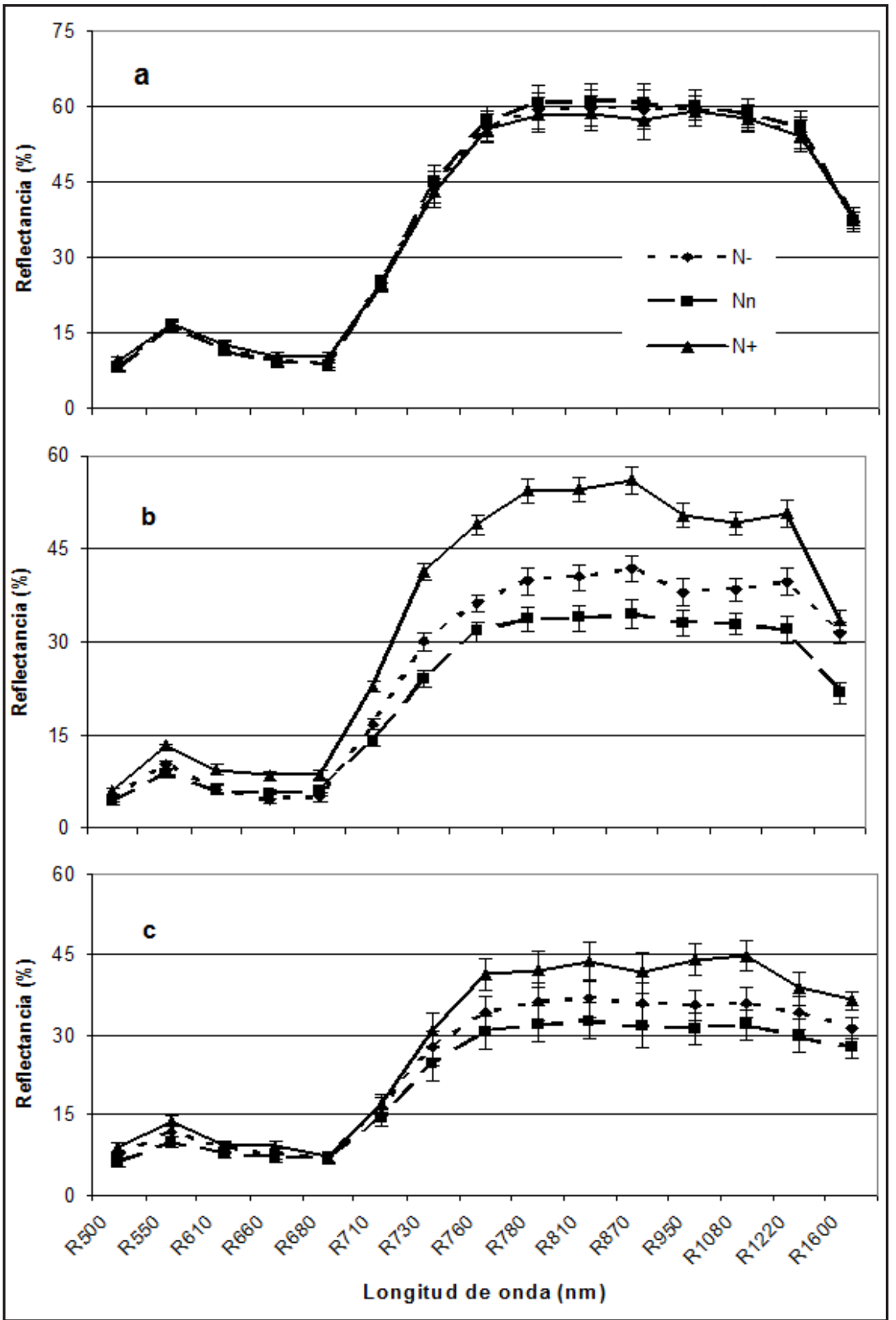

Figura 2. Reflectancia espectral de un cultivo de gerbera para flor de corte cultivada sin suelo en otoño (a), invierno (b) y primavera (c) bajo 3 niveles de fertilización nitrogenada: $\mathrm{N}-=5,6 ; \mathrm{Nn}=9,95 ; \mathrm{N}+=13,6 \mathrm{mmol} / \mathrm{L} \mathrm{N}^{-\mathrm{NO}_{3}}$. Las barras verticales indican DMS (diferencia mínima significativa) a $\mathrm{p} \leq 0,05(\mathrm{n}=15)$ \{spectral reflectance of a gerbera cut flower soilless crop in auntum (a), winter (b) and spring (c) under three levels of nitrogen fertilization: $\mathrm{N}-=5.6 ; \mathrm{Nn}=9.95 ; \mathrm{N}+=13.6 \mathrm{mmol} / \mathrm{L} \mathrm{N}^{-\mathrm{NO}_{3}}$. Vertical bars indicate LSD (least significant difference $)$ at $\mathrm{p} \leq 0.05(\mathrm{n}=15)\}$. Buenos Aires, FAUBA, 2008.

\section{Relación entre IAF y producción de flores}

El gran aumento del área foliar durante el invierno, podría atribuirse a que hay poca producción de flores y los fotoasimilados son direccionados hacia el crecimiento vegetativo, siendo el tratamiento $\mathrm{N}$ - el que muestra mayor tasa de aparición y expansión de hojas nuevas. El nivel de $\mathrm{N}$ inorgánico en la rizosfera gobierna tanto la tasa de crecimiento como el patrón de desarrollo de las plantas superiores. Estas adaptaciones incluyen tanto al metabolismo del nitrógeno propiamente dicho como a múltiples vías metabólicas y expresiones morfogénicas asociadas (Miller et al., 2008).

Una vara floral típica de gerbera incluye un número determinado de hojas maduras, en cuyas axilas hay yemas en dormición. El ápice del rizoma o tallo subterráneo una vez que ha desarrollado una cierta cantidad de hojas se vuelve reproductivo y desarrolla una inflorescencia, al igual que la yema axilar de la hoja más joven (Leffring, 1984), es decir, cada ramificación tiene el potencial para generar dos inflorescencias. Además de la yema axilar de la penúltima hoja desarrollada, nace un nuevo tallo vegetativo que repetirá el ciclo. Frente a este hábito de crecimiento, podría interpretarse que la producción de flores estaría relacionada con la aparición y expansión de hojas, por lo tanto frente a un aumento o disminución del IAF, existirá un efecto sobre la producción, que además será modulado por la variedad y las condiciones ambientales. En este caso se observa que en la primera etapa evaluada correspondiente al periodo otoño, el perfil productivo está relacionado con el IAF, es decir mayor producción en Nn y menor en $\mathrm{N}$ - que presentan el mayor y menor IAF respectivamente. A la salida del invierno, $\mathrm{N}$ - es el tratamiento que mayor IAF alcanza, y es el pico de producción más alto en la primavera, posiblemente por un efecto residual del crecimiento vegetativo (Figuras 1 y 3 ). La medición del IAF podría servir como un índice que permitiría anticiparse a la respuesta productiva del cultivo. Un objetivo de manejo podría ser mantener el IAF dentro de ciertos límites según la estación del año, los cuales de acuerdo con nuestros resultados deberían ser del orden de 3,4 y $2 \mathrm{~m}^{2}$ hoja $/ \mathrm{m}^{2}$ suelo en otoño, invierno y primavera respectivamente.

Efecto de la nutrición nitrogenada en gerbera para flor cortada

Los resultados obtenidos permiten 


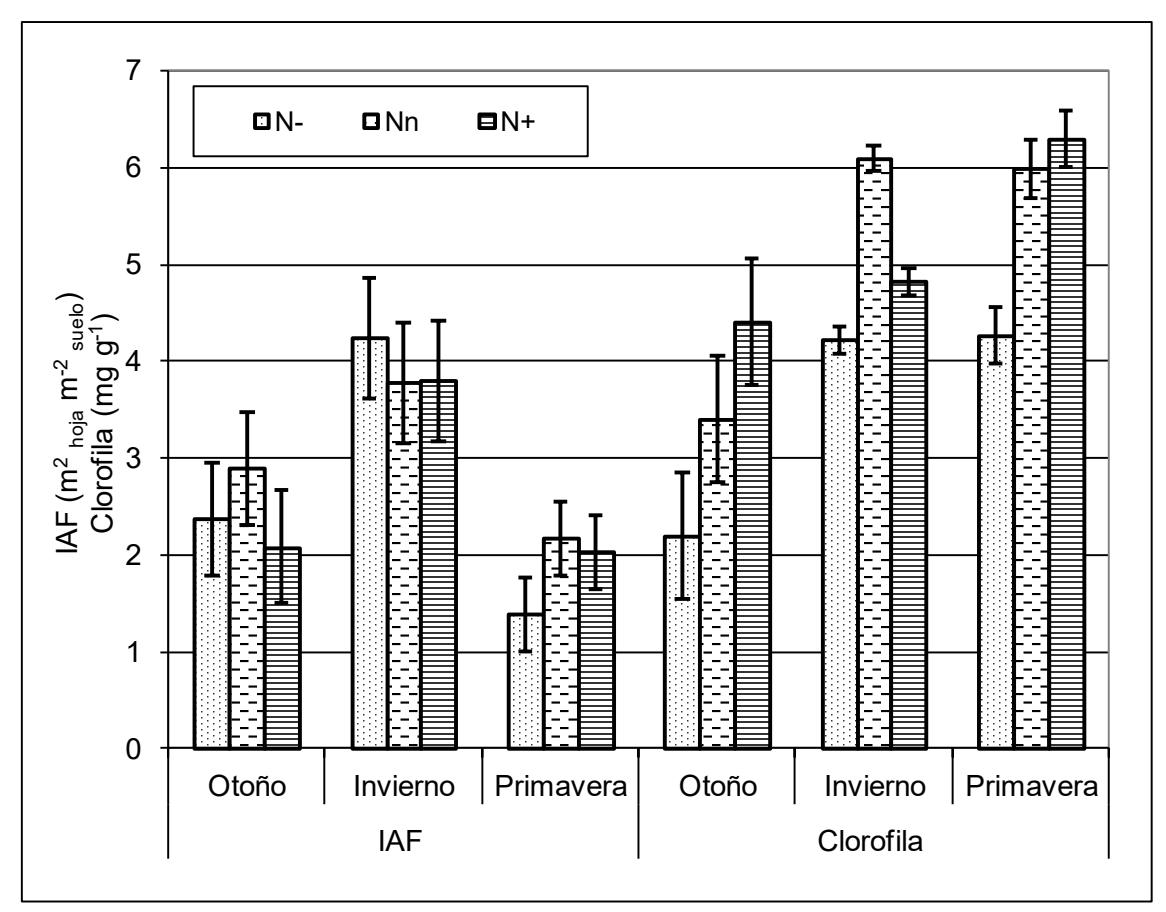

Figura 3. Indice de área foliar y contenido de clorofila en hoja de un cultivo de gerbera para flor de corte cultivada en hidroponía en otoño, invierno y primavera, bajo 3 niveles de fertilización nitrogenada: $\mathrm{N}-=5,6 ; \mathrm{Nn}=9,95 ; \mathrm{N}+=13,6 \mathrm{mmol} / \mathrm{L} \mathrm{N}_{-\mathrm{NO}_{3}}^{-}$. Las barras verticales indican DMS (diferencia mínima significativa) a $\mathrm{p} \leq 0,05(\mathrm{n}=15)$ \{leaf area index and chlorophyll content in leaf of a gerbera cut flower soilless crop in autumn, winter and spring under three levels of nitrogen fertilization: $\mathrm{N}-=5.6 ; \mathrm{Nn}=9.95 ; \mathrm{N}+=13.6 \mathrm{mmol} / \mathrm{L}$ $\mathrm{N}-\mathrm{NO}_{3}$. Vertical bars indicate LSD (least significant difference) at $\left.\mathrm{p} \leq 0.05(\mathrm{n}=15)\right\}$. Buenos Aires, FAUBA, 2008.

inferir que la nutrición nitrogenada en gerbera durante el primer año no modifica sustancialmente la producción total de flores, si se toma el año completo como período de análisis. Esto podría explicarse por el hecho que se trata de una planta perenne, que posee una zona de acumulación de reservas localizada en el rizoma, que serviría de amortiguador frente a variaciones ambientales, resultando poco afectada la actividad reproductiva de la planta. Adicionalmente, se puede considerar que el período de tiempo durante el cual se llevó a cabo el ensayo, corresponde a un tercio de la vida útil de la planta, coincidente con el primer año de producción y por lo tanto con un cultivo joven. Para Dufault et al. (1990) un cultivo de gerbera ya establecido tendría mayores requerimientos nutricionales: mientras que en el primer año la dosis de $\mathrm{N}$ que optimizó productividad y calidad fue de $110 \mathrm{~kg} / \mathrm{ha}$, en el segundo año se necesitó el doble de esta dosis para lograr resultados satisfactorios.
Al analizar períodos mensuales, sí se advierten fuertes variaciones en el rendimiento de gerbera. La fuerte oscilación en la producción de flores en la dosis más alta de $\mathrm{N}$, podría deberse a que un período de alta producción podría significar también imponer alguna limitación a otros componentes de la planta. En Curcuma alismatifolia cv. Gagnep, se comunicó que dosis crecientes de $\mathrm{N}$ desde 0 hasta $50 \mathrm{mg} / \mathrm{L}$ incrementaron la producción de flores en un $330 \%$, pero el crecimiento de raíces y el almacenamiento de reservas resultó deprimido en igual proporción (Ohtake et al., 2006). En nuestro caso, las variaciones observadas en producción de flores podrían tener alguna relación con la acumulación de reservas en raíces, comprometiendo el siguiente ciclo de floración. Así, a un período de gran productividad podría seguir un período de profunda depresión, debido al desbalance nutricional que afecta a la parte subterránea de la planta.

Es probable que de mantenerse las condiciones subóptimas se registren mayores diferencias. Se ha demostrado que plantas de edad avanzada puestas en condiciones adecuadas logran una buena performance productiva, aún mejor que plantas más jóvenes de la misma variedad (Mascarini et al., 2007).

En crisantemo para flor de corte, una alta disponibilidad de $\mathrm{N}$ en la solución nutritiva se asoció con una reducción en el crecimiento de la planta (Barbosa et al., 2000). Tratándose la gerbera de una planta perenne (a diferencia del crisantemo cuyo cultivo es estacional) la alta disponibilidad permanente de $\mathrm{N}$ como nitrato pudo afectar negativamente la productividad en ciertos momentos que serían las fuertes caídas luego de los picos de producción, posiblemente por promover un consumo "de lujo" privilegiando principalmente al crecimiento vegetativo.

Es conocido que la cantidad óptima de $\mathrm{N}$ en la solución nutritiva se relaciona con la disponibilidad de luz y con la temperatura (Carelli et al., 2006), en consecuencia mayor cantidad de $\mathrm{N}$ tiene mayor efecto cuanto más se acerca la estación estival.

El área foliar no muestra diferencias en otoño y primavera, pero disminuye fuertemente del invierno a la primavera en todos los tratamientos, lo cual podría deberse a la removilización de nutrientes debido a la senescencia foliar. El tratamiento $\mathrm{N}$ - es el que más fuertemente disminuye, y en la primavera es significativamente menor que $\mathrm{Nn}$ y $\mathrm{N}+$, y además es el que mayor nivel de producción alcanza en el pico de primavera. Este comportamiento podría estar influenciando el fuerte aumento en la cantidad de flores producidas en la primavera, a expensas de una fuerte reducción del área foliar.

A las dosis ensayadas, si bien se encontraron en algunos momentos diferencias en el IAF y en el contenido de clorofila, una menor dosis de $\mathrm{N}$ no se tradujo totalmente en menor rendimiento, que era lo que se esperaba que ocurriera, mientras que bajo la situación de $\mathrm{N}$ en exceso, se verificó un mayor consumo de $\mathrm{N}$ aunque la productividad no aumentó proporcionalmente, es decir la eficiencia fue menor.

Similares resultados se han obtenido 
al ensayar 4 dosis de $\mathrm{N}$, entre 56 y 112,5 $\mathrm{mg} \mathrm{N} /$ planta/día no encontrando efecto sobre el área foliar, el número de flores producidas ni la calidad de las mismas, a pesar de existir un mayor nivel de fotosíntesis en las plantas con mayor suministro de $\mathrm{N}$, pudiendo relacionarse con un aumento de biomasa vegetativa sin afectar la calidad ni cantidad de flores producidas (Corrales-González et al., 2016).

Jaimez et al. (2013), estudiando la relación entre la tasa fotosintética y la dinámica de producción de flores en ocho cultivares de gerbera, también encontraron diferencias en la fotosísntesis neta que no se tradujeron en diferencias en la productividad, obteniéndose entre 2,5 a 3 flores/ planta/mes; tampoco hubo diferencias significativas en la calidad de flores.

Se concluye que sería posible generar una estrategia de fertilización nitrogenada acorde a cada estación de crecimiento: por ejemplo en primaveraverano se recomendaría una dosis más alta, mientras que en otoño-invierno esta podría reducirse sin un efecto significativo sobre la producción. La reflectancia espectral podría estimar el nivel de $\mathrm{N}$ en riego, queda por mejorar los patrones que permitan predecir de manera rápida y no destructiva, los niveles de suficiencia recomendados, utilizando por ejemplo la estimación de clorofila y el índice de área foliar como variables de control, e incorporando el contenido de $\mathrm{N}$ foliar. Esto permitirá un manejo nutricional más eficiente y a la vez más amigable con el ambiente. Estos modelos deberán ser validados para otras variedades de gerbera y en distintas condiciones ambientales y edad de la planta a fin de generar una herramienta práctica de manejo que pueda utilizarse en cultivos de dos o tres años de edad.

\section{REFERENCIAS}

ATHANASIOU, K; DYSON, BC; WEBSTER, RE; JOHNSON, GN. 2010. Dynamic acclimation of photosynthesis increases plant fitness in changing environments. Plant Physiology 152: 366-373.

BARBOSA, JG; KÄMPF, AN; MARTINEZ,
HEP; KOLLER, OC; BOHNEN, H. 2000. Chrysanthemum cultivation in expanded clay. I. Effect of the nitrogen-phosphorus-potassium ratio in the nutrient solution. Journal of Plant Nutrition 23: 1327-1336.

CARELLI, MLC; FAHL, JI; RAMALHO, JDC. 2006. Aspects of nitrogen metabolism in coffee plants. Brazilian Journal of Plant Physiology 18: 9-21.

CAStillo, ÁR; Ligarreto, GA. 2010. Relación entre nitrógeno foliar y el contenido de clorofila, en maíz asociado con pastos en el Piedemonte Llanero colombiano. Corpoica Ciencia y Tecnología Agropecuaria 11: 122128.

CIEZA, RI. 2014. Caracterización de la producción florícola en el partido de La Plata. Revista de la Facultad de Agronomía 113: 28-37.

CORRALES-GONZÁLES, M; RADA, F; JAIMEZ, R. 2016. Efecto del nitrógeno en los parámetros fotosintéticos y de producción del cultivo de la gerbera (Gerbera jamesonii H. Bolus ex Hook. f.). Acta Agronómica 65: 255-260.

DUFAULT, RJ; PHILLIPS, TL; KELLY, JW. 1990. Nitrogen and potassium fertility and plant populations influence field production of gerbera. HortScience 25: 1599-1602.

FENECH-LARIOS, L; TROYO-DIÉGUEZ, E; TRASVIÑA-CASTRO, M; RUIZESPINOZA, F; BELTRÁN-MORALES, A; MURILLO-AMADOR, B; ZAMORASALGADO, S. 2009. Relación entre un método no destructivo y uno de extracción destructivo, para medir el contenido de clorofila en hojas de plántula de albahaca (Ocimum basilicum L). Universidady Ciencia 25: 99-102.

FERNÁNDEZ-FALCÓN, M; HERNÁNDEZ, M; ALVAREZ, CE; BORGES, AA. 2006. Variation in nutrition along time and relative chlorophyll content of Leucospermum cordifolium cv. 'High Gold' and their relationship with chlorotic sypmptoms. Scientia Horticulturae 107: 373-379.

JAIMEZ, R; ARAQUE, O; ESPINOZA, W; AZOCAR, C. 2013. Dinámica de producción de flores de cultivares de gerbera (Gerbera jamessoni $H$. Bolus): relación con tasas fotosintéticas. Revista de la Facultad de Agronomía de La Universidad del Zulia. 30: 178-191.

JEONG, KY; WHIPKER, B; MCCALL, I; FRANTZ, J. 2009. Gerbera leaf tissue nutrient sufficiency ranges by chronological age. Acta Horticulturae 843: 183-190.

JONES JUNIOR, JB; CASE, VW. 1990. Sampling, handing, and analyzing plant tissue samples. In: WESTERMAN, RL (ed). Soil testing and plant analysis, 3rd ed. Chapter 15. Madison, WI. p. 389-427.

KEDITSU, R. 2013. Gerbera: soil fertility and plant nutrition. Scientific Journal of Agricultural 2: 97-114.

LEFFRING, L. 1984. Influence of temperature on the morphology and flower production of gerbera cultivars. Acta Horticulturae 148:
575-579.

MASCARINI, L, DELFINO, OS, VILELLA, F. 2001. Evapotranspiration of two Gerbera jamesonii cultivars in hydroponics: adjustment of models for greenhouses. Acta Horticulturae 554: 261-269.

MASCARINI, L; LORENZO, G; LEDESMA, D. 2007. Rendimiento y calidad de dos variedades de gerbera en cultivo hidropónico con y sin calefacción del sustrato. In: JORNADAS NACIONALES DE FLORICULTURA, 9. Actas... Argentina: Salta. p. 50-52.

MASCARINI, L; LORENZO, GA; VILELLA, F. 2005. Nitrogen concentration in nutrient solution, post harvest life and flowers commercial quality in hydroponic gerbera. Acta Horticulturae 697: 371-375.

MASCARINI, L; LORENZO, GA; VILLELA, F. 2006. Leaf area index, water index and red:far red ratio calculated by spectral reflectance and its relation with plant architecture and cut rose production. Journal of the American Society of Horticultural Science 131: 313-319.

MILLER, AJ; FAN, X; SHEN, Q; SMITH, SJ. 2008. Amino acids and nitrate as signals for the regulation of nitrogen acquisition. Journal of Experimental Botany 59: 111-119.

OHTAKE, N; RUAMRUNGSRI, S; ITO, S; SUEYOSHI, K; OHYAMA, T; APAVATJRUT, P. 2006. Effect of nitrogen supply on nitrogen and carbohydrate constituent accumulation in rhizomes and storage roots of Curcuma alismatifolia cv. Gagnep. Soil Science and Plant Nutrition 52: 711-716.

PIMPLE, AG; DALAL, SR; NANDRE, DR; GHAWADE, SM; SWARUPA, U. 2006. Yield and quality of gerbera influenced by nitrogen and phosphorus levels under polyhouse conditions. International Journal of Agricultural Science 2: 320-321.

ROGERS, MN; TJIA, BO. 1990. Gerbera production. Portland: Timber Press INC. $116 \mathrm{p}$.

SAHOO, RN; RAY, SS; MANJUNATH, KR. 2015. Hyperspectral remote sensing of agriculture. Current Science 108: 848-859.

SAVVAS, D. 2003. Hydroponics: a modern technology supporting the application of integrated crop management in greenhouse. Food, Agriculture \& Environment 1: 80-86.

SIRIN, U. 2011. Effects of different nutrient solution formulations on yield and cut flower quality of gerbera (Gerbera jamesonii) grown in soilless culture system. African Journal of Agricultural Research 6: 4910-4919.

TREJO-TÉLLEZ, LI; GÓMEZ-MERINO, FC. 2012. Nutrient solutions for hydroponic systems. In: ASAO, T (ed). Hydroponics - A Standard Methodology for Plant Biological Researches. InTech, DOI: 10.5772/37578. Available in: http://www.intechopen.com/ books/hydroponics-a-standard-methodologyfor-plant-biological-researches/nutrientsolutions-for-hydroponic-systems

WALTERS, RG. 2005. Towards an understanding of photosynthetic acclimation. Journal of Experimental Botany 56: 435-447. 